\title{
A New Core Facility For Electron And Ion Microscopy At The University Of Arizona
}

T.J. Zega ${ }^{1,2}$, B.B. Massani ${ }^{3,4}$ Y.J. Chang ${ }^{1,5}$, K. Domanik ${ }^{1}$, K. Nebesny ${ }^{3,4}$, P. Wallace ${ }^{1,5}$, N.R.

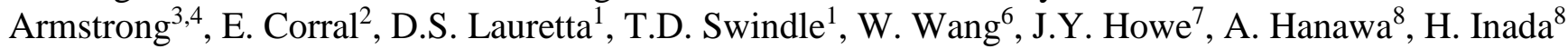

1. Lunar and Planetary Laboratory, University of Arizona, Tucson, U.S.A.

2. Dept. of Materials Science and Engineering, University of Arizona, Tucson, U.S.A.

3. Dept. of Chemistry and Biochemistry, University of Arizona, Tucson, U.S.A.

4. Office of Research, Discovery, and Innovation, University of Arizona, Tucson, U.S.A.

5. Arizona Research Labs, University of Arizona, Tucson, U.S.A.

6. Dept. of Physics, University of Arizona, Tucson, U.S.A.

7. Hitachi High Technologies America Inc., Clarksburg, U.S.A.

${ }^{8 .}$ Hitachi High Technologies Co., Hitachinaka, Japan

Materials research and student training at large "super-land-grant" universities like the University of Arizona (UA) require routine access to state-of-the-art instrumentation that combines 2D- and 3Danalysis of composition and structure from $\mathrm{cm}$ to atomic length scales. Information on the relationship between structure and property is critical to establish the feedback loop between materials characterization, growth, and optimization. The diverse materials-research programs at UA span the sciences and engineering and such programs require a range of spatially resolved analytical tools. Here we describe a core facility for electron and ion microscopy at UA, newly constructed to support these diverse research programs, and provide students, postdocs, and faculty access to state-of-the-art instrumentation and training.

The facility is located in the sub-basement of the Gerard P. Kuiper building for Space Sciences, which was constructed in 1966 with funds provided by NASA. Located approximately $30 \mathrm{ft}$. below ground and slab-on-grade, the basement was renovated and repurposed to house electron and ion microscopes. The Kuiper Core Imaging Facility serves the entire UA community, including regional private- and publicsector users. It is centrally supported by the UA office of Research, Discovery, and Innovation (RDI), as part of an expanding Core Facility program. Professional scientists staff the facility, manage daily operations, train users, and a faculty user committee provides oversight and scientific direction.

The facility consists of parallel instrument bays and includes space for sample preparation, meeting and workshops, and offices for laboratory scientists and visitors (Fig. 1a). A utility corridor was constructed behind several labs to house service equipment for the instruments.

The Scanning Electron Microscope (SEM) suite currently houses two microscopes with room for a third instrument. The Hitachi S-4800 cold-field emission gun (cold FEG) can operate between $0.5 \mathrm{keV}$ and 30 $\mathrm{keV}$. It is equipped with a Thermo-Noran $\mathrm{Si}(\mathrm{Li})$ energy dispersive X-ray spectrometer (EDS) running Noran SystemSix (NSS) software. The Hitachi S-3400 is a W thermal emitter with a variable-pressure chamber and Renishaw InVia Raman system with the Structural and Chemical Analyser (SCA) interface and Reflex microscope. It is equipped with secondary-electron and backscattered-electron detectors and a Thermo-Noran SDD EDS system operating NSS software. The SEM suite also has laboratory benchtop space for sample-preparation and includes a chemical fume hood. See Fig. 1b-d. 
The focused-ion-beam scanning electron microscope (FIB-SEM) lab houses an FEI Helios NanoLab 660. The Helios is equipped with an Elstar electron gun and monochromator, and is capable of electron beam resolution down to $0.6 \mathrm{~nm}$ from $15 \mathrm{kV}$ to $2 \mathrm{kV}$. Its Tomahawk Ga${ }^{+}$ion column can be operated between $65 \mathrm{nA}$ and $500 \mathrm{~V}$ for, respectively, removal of large volumes of material and final sample polishing. Under standard operating conditions, an ion beam resolution $2.5 \mathrm{~nm}$ at $30 \mathrm{kV}$ is achievable. The Helios is equipped with in situ micromanipulation for creation and transfer of lamellae for TEM analysis. It is also equipped with an EDAX EDS system and electron backscatter diffraction analysis system for compositional and crystallographic analysis in two and three dimensions. Multiple polygons are supported for device patterning as well as the ability to directly import customized shapes for patterning or deposition. The Helios is equipped with $\mathrm{C}$ and Pt gas-injection systems. See Fig. 1e.

The Transmission Electron Microscope (TEM) lab houses a newly installed Hitachi HF5000. The HF5000 has a cold FEG and comes with alignments at $200 \mathrm{keV}$ and $60 \mathrm{keV}$. It is equipped with a Hitachi $3^{\text {rd }}$-order spherical aberration corrector for the scanning TEM (STEM) probe. Spectroscopic capabilities include: (1) Oxford Instruments twin silicon-drift detectors (SDD) for EDS providing a total solid angle approaching $2.0 \mathrm{sr}$; and (2) Gatan Quantum Imaging Filter (GIF) for electron energy-loss spectroscopy (EELS). The HF5000 is capable of $\leq 0.4 \mathrm{eV}$ energy resolution for EELS and $78 \mathrm{pm}$ pointto-point resolution in STEM mode for atomic-resolution imaging. See Fig. 1f.

The Electron Microprobe (EMPA) lab houses Cameca SX-50 and Cameca SX-100 Ultra instruments. The SX-50 was installed in 1991 and has been in continuous service for 26 years. It is equipped with a $30 \mathrm{keV} \mathrm{W}$ thermal emission gun, four wavelength dispersive X-ray spectrometers (WDS), 12 diffracting crystals, and a Princeton-Gamma Tech $\mathrm{Si}(\mathrm{Li})$ EDS system, allowing analysis of elements with $\mathrm{Z} \geq 4$. The SX-100 Ultra was installed in 2011 and has been in service for nearly 6 years. It is equipped with a 30 $\mathrm{keV} \mathrm{LaB} 6$ filament, five WDS, 14 diffracting crystals, and an SDD-EDS system, allowing analysis of elements with $\mathrm{Z} \geq 5$. See Fig. $1 \mathrm{~g}$ [1].

\section{References:}

[1] We acknowledge NASA (grants \#NNX12AL47G and \#NNX15AJ22G) and NSF (grants \#1531243 and \#0619599) and the UA RDI for funding of instrumentation. We thank RDI as well as the Lunar and Planetary Laboratory and the UA College of Science for funding the renovation. We thank Charlie Lynn and the UA Office of Planning, Design, and Construction for overseeing all phases of the renovation. We thank Tom Gerbo and Russell Simpson of Sears Gerbo Architecture for design and Richard Burkel and Art Idler of R.W. Burkel Contracting Inc. for managing construction.

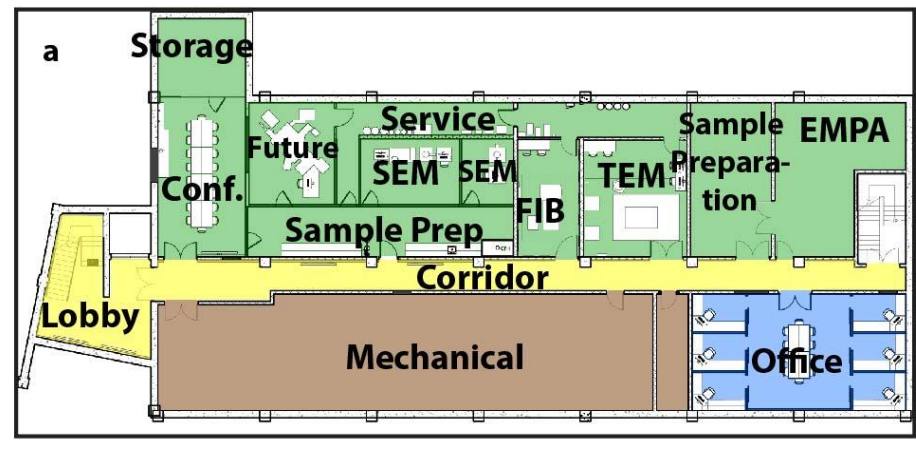

Figure 1. (L) Floor plan of Kuiper Core Imaging Facility.

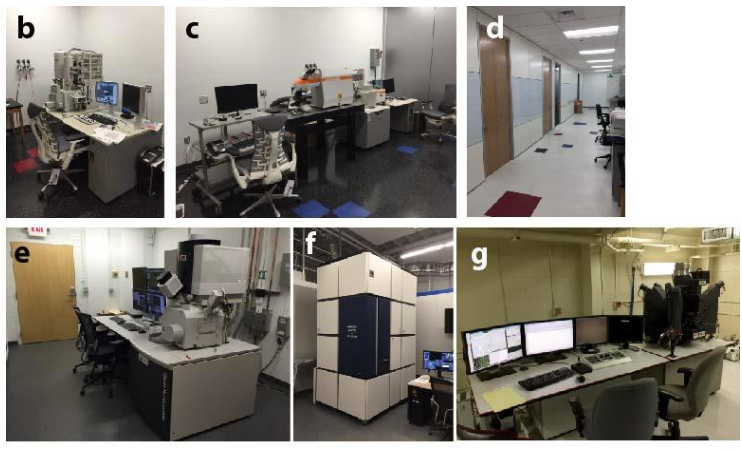

(R) Photographs of laboratories. 